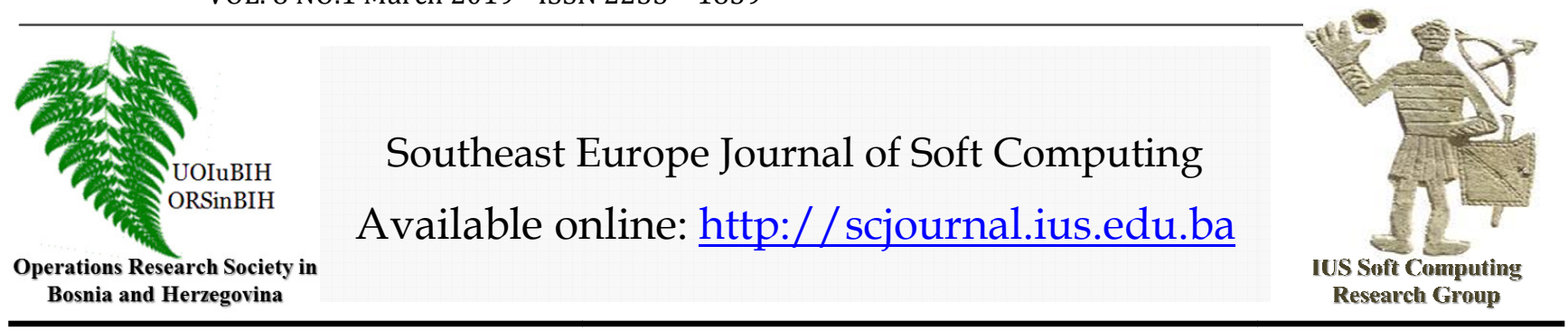

\title{
Transfer Learning Utilization for Banknote Recognition: a Comparative Study Based on Bosnian Currency
}

\author{
Ali Abd Almisreb \\ Faculty of Engineering and Natural Sciences, \\ International University of Sarajevo, \\ Hrasnicka cesta 15, Ilidža 71210 Sarajevo, \\ Bosnia and Herzegovina \\ alimes96@yahoo.com
}

\author{
Mohamed A. Saleh \\ Faculty of Electrical Engineering, \\ Universiti Teknologi MARA (UiTM), \\ Malaysia
}

\section{Article Info}

Article history:

Article received on 13 January 2019 Received in revised form 25 February 2019

Keywords:

Transfer Learning; Deep Learning Convolutional Neural Network; Alexnet; Googlenet; Vgg16; Bosnian Banknotes (BAM)

\begin{abstract}
Transfer learning introduces the ability to perform deep learning models over a small set of data. This paper investigates the utilization of three fine-tuned Convolutional Neural Networks (CNNs), namely, Alexnet, Googlenet, and Vgg16. Alexnet and Googlenet consider as the state-ofthe-art models in deep learning, while Vgg16 preference due to its depth. Each model was fine-tuned, trained, and tested over a dataset contains Bosnian Banknotes (BAM). The dataset covers 11 classes where 10 images were collected through mobile phone camera for each class. Alexnet showed a better performance in terms of completing the training while $\mathrm{Vgg} 16$ showed better performance in terms of accuracy as it achieved $100 \%$ compared to $95.24 \%$ for Alexnet. Googlenet showed less efficient performance by achieving $88.65 \%$.
\end{abstract}

\section{INTRODUCTION}

At present, there are many techniques utilized for image classification, fingerprint-based recognition, iris, and ear recognition, etc. As well known, currency papers are one of the most used medium for circulation, whereas the currency characteristics vary from country to another. Relying on image and features recognition techniques, the researchers investigate and compare different algorithms on their specific identification methods by studying several features of the currency paper such as the color and specific data representation of currency, that lead to finding out a high accurate model for feature extraction and recognition of a specific currency paper.

Recently, deep learning techniques are widely exploited in images recognition. Convolutional Neural Networks $(\mathrm{CNN})$, is one of the popular methods of deep learning which considered as one of the most feasible methods in images recognition field. Since deep learning requires a huge amount of data, the accuracy of the currency recognition algorithm will be dramatically increased by utilizing a big set of data for training. Thus, the recognition will be continuously enhanced based on the highest number of the allocated image for training [1]. There are several approached have been conducted for currency paper 
28 A. A. Almisreb, and M.A. Saleh / Southeast Europe Journal of Soft Computing Vol.8 No.1 March 2019 (27-31)

recognition, such as that was proposed in [2]. This approach based on Ensemble Neural Network (ENN) where each individual network in that ENN was trained using negative correlation learning. It anticipated for solving the problem of currency recognition by applying the proposed approach on dataset includes images of seven types of Bangladeshi currency TAKA. Hence, the images were converted into gray-scale and comprised. Consequently, the pixels of the compressed images used as the input of the proposed network.

In 2008, another Currency detection algorithm was proposed based on compressed gradation [3]. The Artificial Neural Network (ANN) assists in eliminating the noise from the background by implementing a special linear transformation function and ensuring that the image features will not be affected. The threshold value of the grayscale images was set to be in the range between 0 and 125. In order to enhance the robustness for currency detection, an edge detection method considered. Therefore, to achieve that, a set of three-layer backpropagation neural network presented, which effectively detected by different classifications [3]. Hidden Markov Model (HMM) used for currency recognition too. In this method, recognizing the currency paper is no longer limited to the color and the size of the paper but it based on the texture of currency. Therefore, any damage to the currency surface can be overcome. On the other hand, the unclean paper currency could not be recognized [4]. In general, R-CNN composed of several stages, mainly, creating the regions of interest, which are bounding boxes that have a high similarity of containing an interesting object. Next, feature extraction from each region proposal by using a $\mathrm{CNN}$. Another image, which contains a bounding-box, warped in order to match the size of the input layer in the $\mathrm{CNN}$ and then provide it to the next layer. After feature extraction, the features vector fed to support vector machine (SVM) which in turns will provide the final classification decision. R-CNN was trained in multiple stages [5]. Another approach was proposed for Myanmar paper currency recognition [6] where the proposed approach based on the textural feature of the paper currency. The used dataset contains 500 images and classified into 5 classes $(100,200,500,1000$, and 5000) Kyat. Owing to the advantage and the fact that transfer learning does not need a big dataset to perform efficiently, our proposed methodology intended to compare the performance of three deep learning methods, namely, Alexnet, Googlenet and Vgg16 over Bosnian Banknotes.

The rest of the paper organized as follows: Section 2 the recent research in this area, the proposed methodology is explained in Section 3, where the last two sections 4 and 5 demonstrate the achieved results and the conclusion respectively.

\section{RECENT RESEARCH}

The research and development in the area of artificial intelligence made a giant leap in the past few years due to the intensive research and achievements in Deep Learning. Deep learning finds its way to a wide range of applications such as image classification and recognition and object recognition. Currency recognition is one of the targeted applications in deep learning. However, there are several types of research were conducted on paper currency using conventional methods [4], [7]-[9]. Recently, numerous researchers such as [10] commenced using deep learning techniques for paper currency recognition due to the need for recognizing the true and fake paper money. The researcher used Single Shot MultiBox Detector (SSD) which is a pre_trained Convolutional Neural Network (CNN) to detect and recognize New Zealand Dollar (NZD). Another work on Multi-National Banknote achieved by [11]. This study based on Visible-light Line Sensor and CNN. Another study by [12] proposed a MobileNet-based CNN to recognize Bangla Banknote. In the method proposed by [13], Indian Currency Rupee note recognized using a pre_trained CNN. Using Hybrid discriminative models, the study [14] proposed a deep learning model for paper currency recognition and anticounterfeit. Other studies [15], [16] aimed to recognize old and new Ukrainian banknotes using CNN and OpenCV with Keras library. Pham et al. [17] suggested Deep Learning for Banknote Fitness Classification for Korean won (KRW), Indian rupee (INR), and Unites States dollar (USD). They used Reflection Images by a Visible-Light One-Dimensional Line Image Sensor. Therefore, the Deep Learning applications for paper currency and Banknotes recognition and classification considered as one of the promised techniques in that area.

\section{METHODOLOGY}

The method for conducting this study is mainly based on two phases; first one is dataset collection for the Bosnian Currency paper, while the second phase is dedicated for testing and training that dataset on Alexnet, googlenet and Vgg16 pre_trained CNNs models.

\subsection{Paper Currency Acquisition}

A designed dataset consists of 110 images used to train and test each of the fine-tuned CNNs, i.e. Alexnet, Googlenet, and Vgg16. The dataset contains images of the front and the back sides of the following Bosnian currency notes: 5, 10,20 and 50 cents in addition to 1, 2, 5, 10, 20, 50 and 100 Bosnian Mark (KM) which sometimes known as BAM. 10 images collected of each class. Figure 1 shows samples of the dataset. 


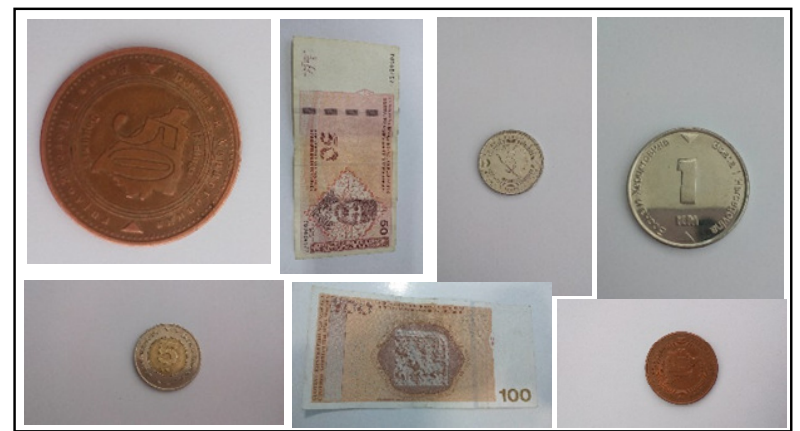

Figure 1: Samples of the dataset

\subsection{CNN Models}

A Convolutional Neural Network (CNN) models comprise different types of multi-layer neural networks, dedicated to recognizing the visual features of images utilizing the structure of their pixels. The modern and commonly used CNN models are AlexNet, VGG16/19, GoogLeNet, and ResNet. In this section, the most relevant models to currency recognition, AlexNet, GoogLeNet, and VGG 16.

\subsubsection{Alexnet}

Alexnet is a Convolutional Neural Network designed by [18]. This CNN is considered as the most studied CNN [19] because of its suitability tradeoffs between speed and accuracy [19]. It contains 8 learned layers, 5 convolutional layers, and 3 fully-connected layers. The last fullyconnected layer connects to 1000 classes and the rest of the network is considered as a feature extractor. AlexNet can yield 4096-dimensional feature vector for each image, which contains the activations of the hidden layer immediately prior to the output layer. This network accepts an input image of size $227 \times 227 \times 3$ as a requirement of the input image layer.

\subsubsection{Googlenet}

Googlenet is the winner of the ILSVRC competition held in 2014 by Google. It is a convolutional neural network architecture implementing a deep module called the inception as described in [20] with 22 layers. Googlenet accepts input images with size $224 \times 224 \times 3$.

\subsubsection{Vgg16}

Vgg16 model improved its architecture over Alexnet by using multiple $3 \times 3$ kernel-sized filters instead of the 11 and 5 kernel-sized filters employed by Alexnet in the first and second convolutional layers. With smaller filters, the depth of the Vgg16 model increasing enabled it to learn more complicated features. The width (the number of layers) of convolutional layers in $\mathrm{Vgg}$ models is comparably small, beginning from 64 in the first layer and increased by a factor of 2 after each max-pooling, reaching 512 at the final convolutional layer. In this paper, Vgg16 selected as the models for banknote recognition simply because it has the deepest number of layers. The network has 41 layers, and there are 16 layers with learnable weights: 13 convolutional layers, and 3 fully connected layers [21]. Vgg16 accepts images with a size of $224 \times 224 \times 3$.

\subsubsection{Fine-tuning}

The last three layers of the pre-trained CNNs are retained as a fixed feature extractor for our dataset. The first step in transfer learning is specifically for replacing the last three layers in the pre-trained $\mathrm{CNN}$ with a set of layers that can classify our targeted 11 classes.

\section{RESULTS}

This section provides details about training and testing the three fine-tuned models. First, Figure 2 shows the performance-based accuracy for training Vgg16, Alexnet, and Googlenet respectively. It is clearly shown that Vgg16 demonstrate the utmost performance compare to Alexnet and Googlenet. In contrast, Vgg16 required a longer time to complete the training. In terms of learning quickness, Googlenet appears to be faster than Alexnet and Vgg16 as illustrated in Figure 3. This figure gives more details about the fine-tuned CNNs loss function.

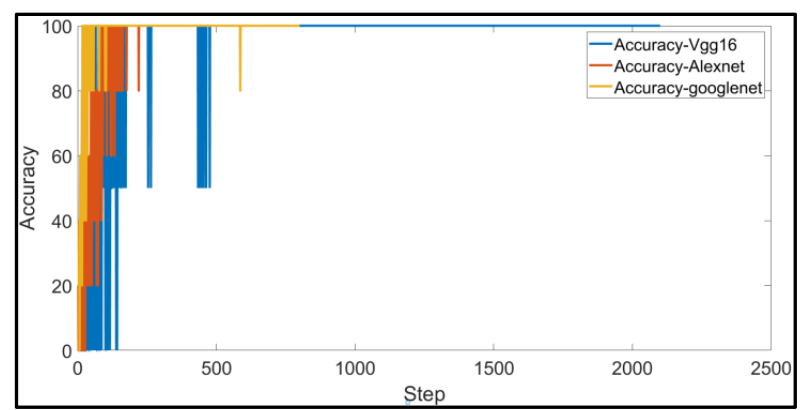

Figure 2: Accuracy of Vgg16, Alexnet, and Googlenet

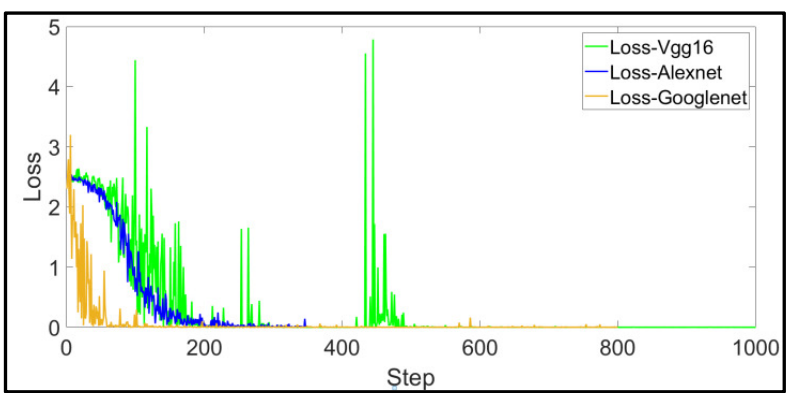

Figure 3: Loss function of Vgg16, Alexnet, and Googlenet 
In order to provide more details about the performance of the finely-tuned networks, we provide the confusion matrixes of each CNN. Figure 4 shows that Alexnet confused by class 5 and 10, Googlenet shows less efficiency as it confused by more classes, namely class 2 , 4, 8, 10 and 12. Figure 5 shows the performance of the Googlenet training. Eventually, Vgg16 shows the best performance compare to Alexnet and Googlenet as Figure 6 where it achieved $100 \%$ accuracy.

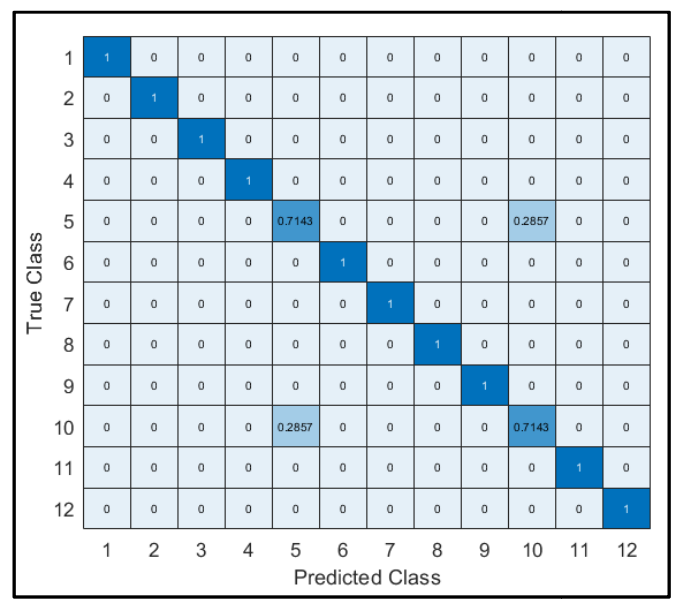

Figure 4: Confusion matrix of Alexnet

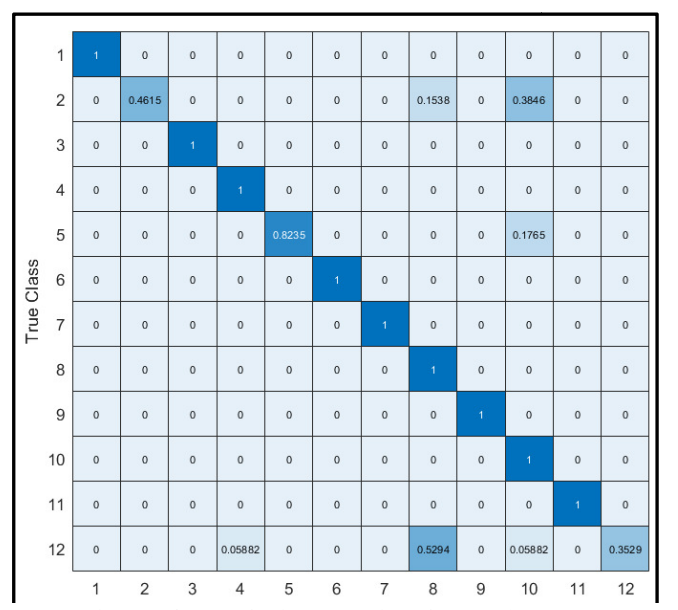

Figure 5: Confusion matrix of Googlenet

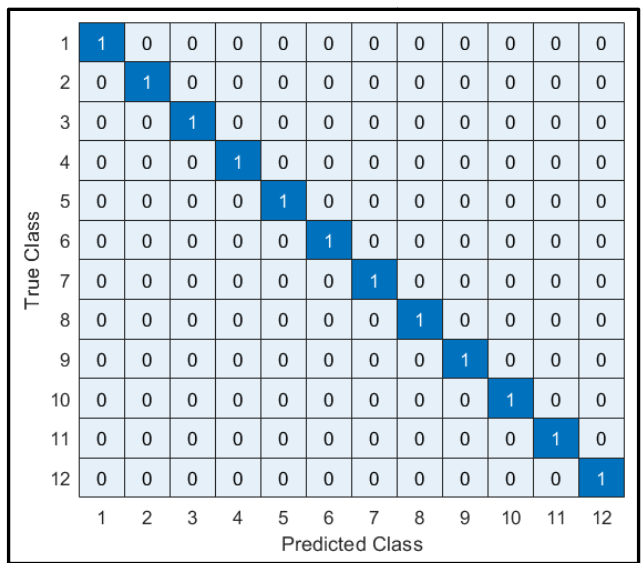

Figure 6: Confusion matrix of Vgg16

\section{CONCLUSION}

This paper provided an explanation of the utilization of the concept Transfer Learning for Banknote recognition. Bosnian currency papers dataset has been used for train and test three fine-tuned Deep Learning models, namely Alexnet, Googlenet, and Vgg16. The later model, Vgg16 showed an outstanding performance compared to Alexnet and Googlenet. Whereas the accuracy achieved for $\operatorname{Vgg} 16$ is $100 \%$, while it is $95.24 \%$ and $88.65 \%$ for Alexnet and Googlenet respectively.

\section{REFERENCES}

[1] R. Wu, S. Yan, Y. Shan, Q. Dang, and G. Sun, "Deep Image: Scaling up Image Recognition," 2015.

[2] K. K. Debnath, S. U. Ahmed, Shahjahan, and K. Murase, "A paper currency recognition system using negatively correlated neural network ensemble," J. Multimed., 2010.

[3] D. A. K. S. Gunaratna, N. D. Kodikara, and H. L. Premaratne, "ANN Based Currency Recognition System using Compressed Gray Scale and Application for Sri Lankan Currency Notes SLCRec," Eng. Technol., vol. 2, no. 9, pp. 235240, 2008.

[4] H. Hassanpour and P. M. Farahabadi, "Using Hidden Markov Models for paper currency recognition," Expert Syst. Appl., 2009.

[5] R. Girshick, "Fast R-CNN," in Proceedings of the IEEE International Conference on Computer Vision, 2015.

[6] K. N. N. Hlaing and A. K. Gopalakrishnan, "Myanmar paper currency recognition using GLCM and k-NN," in Second Asian Conference on Defence Technology (ACDT), 2016, pp. 67-72. 
31 A. A. Almisreb, and M.A. Saleh / Southeast Europe Journal of Soft Computing Vol.8 No.1 March 2019 (27-31)

[7] V. Abburu, S. Gupta, S. R. Rimitha, M. Mulimani, and S. G. Koolagudi, "Currency recognition system using image processing," in 2017 10th International Conference on Contemporary Computing, IC3 2017, 2018.

[8] C. Bhurke, M. Sirdeshmukh, and P. M. S. Kanitkar, "Currency Recognition Using Image Processing," Int. J. Innov. Res. Comput. Commun. Eng., 2015.

[9] H. Hassanpour, A. Yaseri, and G. Ardeshiri, "Feature extraction for paper currency recognition," in 2007 9th International Symposium on Signal Processing and its Applications, ISSPA 2007, Proceedings, 2007.

[10] Q. Zhang and W. Q. Yan, "Currency Detection and Recognition Based on Deep Learning,” 2019.

[11] T. D. Pham, D. E. Lee, and K. R. Park, "Multinational banknote classification based on visiblelight line sensor and convolutional neural network," Sensors (Switzerland), vol. 17, no. 7, 2017.

[12] H. Murad, N. I. Tripto, and M. E. Ali, "Developing a bangla currency recognizer for visually impaired people," 2019.

[13] S. Mittal and S. Mittal, "Indian Banknote Recognition using Convolutional Neural Network," in Proceedings - 2018 3rd International Conference On Internet of Things: Smart Innovation and Usages, IoT-SIU 2018, 2018.

[14] V. D. Hoang and H. T. Vo, "Hybrid discriminative models for banknote recognition and anticounterfeit," in NICS 2018 - Proceedings of 2018 5th NAFOSTED Conference on Information and Computer Science, 2019.

[15] Y. Liu, J. He, and M. Li, "New and Old Banknote Recognition Based on Convolutional Neural Network," 2018.

[16] M. Jiao, J. He, and B. Zhang, "Folding Paper Currency Recognition and Research Based on Convolution Neural Network," in 2018 International Conference on Advances in Computing, Communications and Informatics, ICACCI 2018, 2018.

[17] T. D. Pham, D. T. Nguyen, W. Kim, S. H. Park, and K. R. Park, "Deep learning-based banknote fitness classification using the reflection images by a visible-light one-dimensional line image sensor," Sensors (Switzerland), 2018.

[18] A. Krizhevsky, I. Sutskever, and G. E. Hinton, "ImageNet Classification with Deep Convolutional Neural Networks," Adv. Neural Inf. Process. Syst., pp. 1-9, 2012.

[19] H. C. Shin et al., "Deep Convolutional Neural Networks for Computer-Aided Detection: CNN Architectures, Dataset Characteristics and Transfer Learning," IEEE Trans. Med. Imaging, 2016.

[20] C. Szegedy et al., "Going deeper with convolutions," Proc. IEEE Comput. Soc. Conf. Comput. Vis. Pattern Recognit., vol. 07-12-June, pp. 1-9, 2015.

[21] K. Simonyan and A. Zisserman, "Very Deep Convolutional Networks for Large-Scale Image Recognition," Int. Conf. Learn. Represent., 2015. 